\title{
The contribution of halo red giant mass loss to the high-velocity gas falling onto the Milky Way disk
}

\author{
K. S. de Boer ${ }^{\star}$ \\ Sternwarte, Universität Bonn, Auf dem Hügel 71, 53121 Bonn, Germany \\ Received 24 December 2003 / Accepted 10 February 2004

\begin{abstract}
The origin of gas falling from the halo toward the disk of the Milky Way is still largely unclear. Here the amount of gas shed by the (older) halo red giants is estimated. The distribution of red giants (RGs) in the halo is not known but that of a subset of stars in the post RG phase, the sdB stars of the horizontal-branch (HB), is. Using the mid-plane density and $z$-distribution of sdB stars, the ratio of sdB stars to all $\mathrm{HB}$ stars, and the RG mass loss, the infall due to total mass lost by all halo RG stars at $z>1 \mathrm{kpc}$ is calculated. For the extended halo component $\dot{M}_{\text {halo RGs }} \simeq 1.4 \times 10^{-5} M_{\odot} \mathrm{kpc}^{-2} \mathrm{yr}^{-1}$ while the thick disk component RGs contribute $\dot{M}_{\text {thick disk RGs }} \simeq 5.4 \times 10^{-5} M_{\odot} \mathrm{kpc}^{-2} \mathrm{yr}^{-1}$, each with an uncertainty of a factor 4 . The total rate of infall due to RG mass-loss is $\dot{M}_{\mathrm{RGs}}$ at $z>1 \mathrm{kpc} \simeq 7 \times 10^{-5} M_{\odot} \mathrm{kpc}^{-2} \mathrm{yr}^{-1}$, a sizeable fraction of the equally uncertain observed rate of infall of material. Since most of the RG stars in the extended halo are old, their mass loss is predominantly metal-poor, while that of the disk RGs is more metal-rich. The galactic fountain flow provides additional metal-rich infall and small galaxies being accreted contribute to the infall of gas as well.
\end{abstract}

Key words. Galaxy: halo - Galaxy: structure - Galaxy: evolution

\section{The infall problem}

The detection at high galactic latitude of complexes of neutral hydrogen gas having their velocity directed toward the Solar vicinity (Muller et al. 1963) showed that neutral gas clouds are falling toward the Milky Way disk. It was speculated that the gas might come from intergalactic space (Oort 1966), in which case it should be almost free of metals. The discovery of this influx of possibly pristine matter was embraced by modellers of the evolution of the Milky Way. The "closed box" evolution models predicted many more metal-poor G-dwarfs than actually are known to exist today (see reviews by Audouze \& Tinsley 1976 or Pagel 1997). Since that time the amount of infall needed in the evolution models was taken either as a free parameter or set equal to the amount of infall as can be estimated from the observed halo high-velocity clouds (HVCs).

That rate of infall observed was rather uncertain because in the 1970s only part of the sky had been surveyed for H I. In addition, the distance to the HVCs as well as intermediate velocity clouds (IVCs) was unknown (and is still largely unknown today; see review by Wakker \& van Woerden 1997). Knowledge of such distances is, of course, crucial for the calculation of the mass in HVCs and IVCs and thus of an infall rate.

\footnotetext{
* e-mail: deboer@astro.uni-bonn.de
}

The infalling material ${ }^{1}$ turned out to contain metals, a fact clearly demonstrated by observations with the IUE, HST, ORFEUS, and FUSE spectrographs (see, e.g., Savage \& de Boer 1981; Savage \& Sembach 1996; Richter et al. 2001; Collins et al. 2003). Yet the metal content varies from close to Solar to $1 / 10$ or less (compare Richter et al. 1999 with Wakker et al. 1999). For a full list of IVCs and HVCs detected in metal absorption lines see Wakker (2001).

These findings fueled the debate about the origin of the infalling gas. The models developed can be grouped into three categories. One category has the clouds as the cooling part of a galactic fountain-like flow (Shapiro \& Field 1976; Bregman 1980) and models for the kinematics of this flow show broad consistency with observed velocities (Bregman 1980; Kaelble et al. 1985; Wakker 1991). Another group sees some of the HVCs as material falling in from intergalactic space (Oort 1966, 1970), possibly even gas from within the Local Group (Blitz et al. 1999). In addition there is the gas of the Megallanic Stream, clearly strewn along the path of the Magellanic Clouds through the Milky Way halo, which falls toward the disk. Also

\footnotetext{
${ }^{1}$ Essentially all gas seen at high galactic latitudes can be regarded as falling toward the Milky Way disk; to see that one has to correct each observed velocity for the velocity of the Sun in its orbit around the Milky Way. Moreover, any gas at large $z$-distance (thus being halo gas) having zero vertical velocity would blend in velocity with gas of the disk near the Sun. For more on the problem see, e.g., Kaelble et al. (1985).
} 
gas from other caught satellite dwarf galaxies (cf., Helmi \& White 1999) may be present.

Also molecular hydrogen was detected in HVCs and IVCs (see Richter et al. 1999; Gringel et al. 2000; Bluhm et al. 2001; Richter et al. 2001), which would require the presence of dust. Recently, Evans et al. (2003) discovered dust in the globular cluster NGC 7078. This material will stay in the cluster until it can be stripped when the globular cluster on its orbit zippes through the Milky Way disk. Halo RG stars, however, would shed their gas and dust right into the halo.

The amount of gas shed by red-giant (RG) stars in the halo of the Milky Way (MW) is proposed to be an essential component contributing to the infall. Its production rate is assessed, to be compared to the observational facts about infall available today.

\section{Age, mass, and metallicity of halo stars}

The MW halo is composed of stars belonging to the MW ab initio, most likely supplemented by stars accreted from satellite objects (e.g., the Sagittarius dwarf galaxy).

$\mathrm{Ab}$ initio halo stars are as old as the globular clusters ( $\simeq 13$ Gyr). They likely have a chemical composition similar to that of globular cluster stars. The main-sequence mass $M_{\text {init }}$ of those stars being now RG was, assuming they are metalpoor $(Z=0.001)$, between 0.92 and $0.82 M_{\odot}$ (see the evolution tracks by Schaller et al. 1992).

RG stars belonging to the MW thick disk will in part be younger and more metal-rich than the ab inito halo stars. Their $M_{\text {init }}$ is larger, up to $1.5 M_{\odot}$ (or just up to $1.1 M_{\odot}$ for solar metallicity, $Z=0.02$; Schaller et al. 1992).

It has been speculated that some young stars are present in the halo. In high latitude blue star surveys stars were found that seemed to have (from Balmer line fits) main-sequencelike gravities. If these were indeed main-sequence stars, then one would have star formation in the halo. However, most such claims did not stand up to scrutiny. Their number is small anyway and their effect on overall halo star mass loss is negligible.

Accretion of stars from satellite galaxies took place in the early phases of the MW (see the review by Freeman \& Bland-Hawthorne 2002). Those accreted satellites were rather metal-poor. Due to their disruption no new stars have formed in the debris since accretion so that their RGs have $M_{\text {init }}$ in the same range as those proper of the MW halo. However, more recent accretion may have contributed more massive stars, but their fraction of the total halo mass is likely very small and so will be ignored.

\section{Calculating the mass lost by halo Red Giants}

To calculate the total mass lost by RG stars, the mass loss, the spatial distribution of RGs in the halo, and the total number of RGs must be known. The RG star distribution is, however, essentially unknown and therefore must be derived in an indirect manner. The sdB stars serve the purpose. They are a subclass of HB stars and HB stars have RG stars as progenitors. The various parameters needed for the study are described in the following subsections. The RG mass loss will be discussed in
Sect. 3.1, the spatial distribution of $\mathrm{sdB}$ stars is described in Sect. 3.2 while the ratio of $\mathrm{sdB}$ stars to all $\mathrm{HB}$ stars is discussed in Sect. 3.3. Finally, the mean RG mass loss rate is calculated in Sect. 3.4 and the total mass lost is calculated in Sect. 3.5.

\subsection{Mass lost by one RG star}

Red giants evolve and lose mass and, if $M_{\text {init }}<1.5 M_{\odot}$, end up as horizontal-branch (HB) stars. HB stars are well defined objects in the core He burning phase having a He core of $\simeq 0.5 M_{\odot}$ surrounded by a hydrogen shell ranging from at most $0.02 M_{\odot}$ (the very blue $\mathrm{sdB}$ and the BHB stars) via the HBA and RR Lyr stars to those with a shell of up to $0.4 M_{\odot}$ (the red HB (RHB) stars). The end product of the evolution of RGs is thus an HB star with $M_{\text {end }} \simeq 0.5 M_{\odot}$ (BHB star) to $M_{\text {end }} \simeq 0.9 M_{\odot}$ (RHB star). For the (old) halo RGs $M_{\text {init }}<1.0 M_{\odot}$.

Combining the values given above for $M_{\text {init }}$ and $M_{\text {end }}$ (the lowest $M_{\text {init }}$ and the highest $M_{\mathrm{HB}}: 0.82$ and $0.9 M_{\odot}$, respectively; the highest $M_{\text {init }}$ and the lowest $M_{\mathrm{HB}}: 1.0$ and $0.55 M_{\odot}$ ) shows that the total range possible for the amount lost by halo RGs is $M_{\text {lost }}=0.0$ to $0.45 M_{\odot}$. A reasonable mean for the mass lost by metal-poor stars is

$\overline{M_{\text {lost }}}=M_{\text {init }}-M_{\text {end }} \simeq 0.3\left[M_{\odot}\right]$ per star.

\subsection{Number and distribution of halo HB stars}

Several studies exist about the distribution of stars in the halo. Following earlier work, Chiba \& Beers (2000) derived equidensity contours for the halo distribution from the kinematics of solar neighbourhood metal-poor $([\mathrm{Fe} / \mathrm{H}]<-1)$ stars. One important result from their work is that the density distribution in the halo of the subsample with $-1.6<[\mathrm{Fe} / \mathrm{H}]<-1.0$ is not very different from that of the poorest subsample $([\mathrm{Fe} / \mathrm{H}]<$ -1.8 ), albeit somewhat more flattened. Chiba \& Beers give diagrams with the spatial distribution of stars in $R$ and $z$. Their distribution can, in the solar neighbourhood, be represented by an exponential with $h_{z} \simeq 20 \mathrm{kpc}$

We need, however, to know the distribution of just the RG stars. This is observationally difficult mostly because RGs cover such a large range in $M_{V}$. Stars in the next evolutionary stage, the HB stars, are representative for the RG distribution. Of those, the distribution of the sdB stars is well studied. We need to know the midplane density and the vertical distribution.

Several studies exist aiming at finding the spatial distribution of sdB stars perpendicular to the MW dsik. In one study, the disk region was searched for these stars to arrive at a space density of $n(0)_{\text {disk sdB }} \simeq 2 \times 10^{-6} \mathrm{pc}^{-3}$ (Downes 1986). Other studies explored well defined high-latitude fields, attempting to obtain a "complete sample" of sdB stars out to some distance and then fit a vertical distribution function (mostly an exponential) to arrive at a mid-plane density and a scale height. Examples of such studies are Heber (1986) and Moehler et al. (1990). These led to scale heights of $h_{\mathrm{sdB}} \simeq 200 \mathrm{pc}$. Villeneuve et al. (1995) used an all sky sample and a " $V / V_{\mathrm{m}}$ test" (Schmidt 1968), arriving at mid-plane densities and scale heights as well. A thorough appraisal of such work and earlier studies was made by Villeneuve et al. (1995), concluding 
that $n(0)_{\text {disk sdB }}=3( \pm 1) \times 10^{-7} \mathrm{pc}^{-3}$ and $h_{\mathrm{sdB}}=600( \pm 200) \mathrm{pc}$. Their midplane density will be used below.

Not affected by problems of completeness of those studies is the derivation of the scale height from the $z$-distance statistics of a large sample of galactic orbits (calculated using a galactic potental). In this manner, Altmann et al. (2004) arrived at the existence of two populations of sdB stars, a halo one with $h_{\text {halo } \mathrm{sdB}} \simeq 7 \mathrm{kpc}$ and a disk one with $h_{\text {disk sdB }} \simeq 0.9 \mathrm{kpc}$. No absolute midplane density can be derived from their data, but they estimate that the midplane density ratio of halo and thick disk sdBs is $n(0)_{\text {halo } \mathrm{sdB}}=0.0125( \pm 25 \%) \times n(0)_{\text {disk sdB }}$.

Note that on the direct observational side, an irregular halo $\mathrm{HB}$ star distribution out to $45 \mathrm{kpc}$ is seen in the SDSS data (Yanny et al. 2000).

From these parameters one can calculate the number ratio of sdB stars in each of these populations. Integration over the exponential distributions leads to

$N_{\text {halo sdBs }} / N_{\text {disk sdBs }}=\frac{n(0)_{\text {halo sdB }} \cdot h_{\text {(halo sdB })}}{n(0)_{\text {disk sdB }} \cdot h_{(\text {disk sdB })}}$

so that, with the numbers of Altmann et al. (2004), the total number ratio of halo to disk sdB stars is 0.07 .

Combining the Villeneuve et al. (1995) mid-plane density with the halo to disk ratio from Altmann et al. leads to $n(0)_{\text {halo sdB }}=3.7 \times 10^{-9} \mathrm{pc}^{-3}=3.7 \mathrm{kpc}^{-3}$.

The total number of sdB stars in the halo follows from integration over the $z$-distribution $N=\int_{z_{\mathrm{b}}}^{z_{\mathrm{t}}} n(0) \mathrm{e}^{-z / h_{z}} \mathrm{~d} z$, with $z_{\mathrm{b}}$ and $z_{t}$ the bottom and the top of the layer of integration. Thus

$N_{\mathrm{sdB}}=n(0)_{\mathrm{sdB}} h_{z}\left(\mathrm{e}^{-z_{\mathrm{b}} / h_{z}}-\mathrm{e}^{-z_{\mathrm{t}} / h_{z}}\right)=n(0)_{\mathrm{sdB}} h_{z} g$

in which $g$ is the integral's value.

For the current analysis the rather well-defined Villeneuve et al. (1995) mid-plane density, the ratio of the space density of halo to disk sdB stars, and the respective scale heights will be adopted to represent the extended halo RG distribution. Both the halo and thick disk components are considered. For the thick disk $h_{z}=0.9 \mathrm{kpc}$ from Altmann et al. (2004) and $n(0)_{\text {disk sdB }}=3 \times 10^{2} \mathrm{kpc}^{-3}$ from Villeneuve et al. (1995). For the halo the values $h_{z}=7 \mathrm{kpc}$ from Altmann et al. (2004) and $n(0)_{\text {halo sdB }}=3.7 \mathrm{kpc}^{-3}$ (see above) will be used. The integration is from $z=1 \mathrm{kpc}$ to infinity and $g$ thus gives the fraction of stars of each population above $z=1 \mathrm{kpc}$. For the halo sdBs $g=0.87$ and for the (thick) disk sdBs $g=0.33$.

\subsection{The ratio of $s d B$ stars to all HB stars}

A further important parameter to estimate is the number ratio of sdB stars to all $\mathrm{HB}$ stars $f_{\mathrm{all}} \mathrm{HB} / \mathrm{sdB}=n_{\mathrm{all} \mathrm{HB}} / n_{\mathrm{sdB}}$.

One can look at CMDs of globular clusters and count the blue HB stars (within some colour range) in relation to the total number of HB stars. Here the problem is to define which globular cluster would be typical for the halo population. Such a cluster does not exist. Alternatively, one could add up the CMDs of several globular clusters each representing some part of the history and characteristics of the halo population. Such a procedure leads to a ratio of blue HB stars to all HB stars of crudely a factor of 10. However, the sdB stars are in CMDs rather on the "vertical part" of the HB, and this colour and brightness range is observationally still poorly sampled (but see, e.g., Rosenberg et al. 2000).

A different approach is to relate the so-called "birth rate" of sdB stars in the MW disk to that of, e.g., the White Dwarfs. The birth rate is in a steady state the ratio of the observed space density divided by the phase life of such stars. For the sdB phase life one can use $t_{\mathrm{HB}}$, since the phase life of all HB stars covers the rather small range of 0.8 to $1.2 \times 10^{8} \mathrm{yr}$ for all types (see Charbonnel et al. 1996). Comparing the sdB star birth rate, $n_{\mathrm{sdB}} / t_{\mathrm{HB}}$, with that of the WDs, Villeneuve et al. (1995) find that the sdB stars make up just about $1 \%$ of all stars becoming WD. Since WDs are the end product only of those stars that go through the HB phase, the number looked for, the number ratio of sdB stars to all HB stars, is

$f_{\text {all } \mathrm{HB} / \mathrm{sdB}}=n_{\mathrm{all} \mathrm{HB}} / n_{\mathrm{sdB}}=100$.

The two approaches chosen differ in result by a factor of 10 . The number through the birth rate is, given the method of derivation, much more likely to be accurate.

\subsection{Mass loss rate of one halo RG star}

To calculate the total mass lost by the RG halo stars per unit of time one may attempt to define the length of the RG mass loss phase. Noticeable RG mass loss takes place only in the last $1 \%$ of the evolution time from the main sequence up to the Heflash (end of the RG phase) according to the models of Schaller et al. (1992) and Charbonnel et al. (1996). This slow mass loss amounts to about $10 \% M_{\text {init }}$. However, the bulk of the mass loss occurs at the tip of the red giant branch and is episodic (Origlia et al. 2002), mostly in relation to the He flash. Defining an average all halo RG mass loss rate with little knowledge about the RG mass loss process is nigh to impossible.

A much simpler approach is to assume that also the population of the stellar halo is in a steady state, i.e., the number of HB stars currently existing is representative of the number of HB stars over a considerable time. The phase life of HB stars, $t_{\mathrm{HB}}$ (the phase of core-He burning), is well known. As mentioned above, it is 0.8 to $1.2 \times 10^{8}$ y (Charbonnel et al. 1996). The shorter time in this range is for metal poor stars, the longer for solar metallicity stars. Thus each HB star (all of them have been RG star) represents an RG star with its mass shed.

The spatially and time averaged mass loss rate of one RG star thus must be

$\overline{\dot{M}_{\text {one RG }}}=\overline{M_{\text {lost }}} / t_{\mathrm{HB}} \simeq 0.3 / 10^{8} \quad M_{\odot} \mathrm{yr}^{-1}$,

with $\overline{M_{\text {lost }}}$ from Eq. (1) and $t_{\mathrm{HB}}$ from above.

\subsection{The total halo RG mass loss and infall rate}

The total RG mass-loss rate projected onto a unit area of the Milky Way disk from one hemisphere follows combining Eqs. (3)-(5) given above into $\dot{M}_{\text {halo RGs }}=N_{\mathrm{sdB}} \times f_{\text {all HB } / \mathrm{sdB}} \times$ $\overline{\dot{M}_{\text {one RG }}} \quad M_{\odot} \mathrm{kpc}^{-2} \mathrm{yr}^{-1}$. This mass loss is calculated for the halo and thick-disk components separately. To account for both 
hemispheres, its value has to be multiplied by a factor of 2 so that the total RG mass-loss rate projected onto MW disk

$\dot{M}_{\mathrm{RGs}}=2 \times n_{0 \mathrm{sdB}} \times h_{z} \times g \times f_{\mathrm{all} \mathrm{HB} / \mathrm{sdB}} \times \overline{M_{\mathrm{lost}}} / t_{\mathrm{HB}}$

in units of $M_{\odot} \mathrm{kpc}^{-2} \mathrm{yr}^{-1}$.

\subsubsection{Extended Halo RG mass loss}

For the extended halo component the values determined above, using $f_{\text {all HB } / \mathrm{sdB}}=100$, lead with Eq. (6) to $\dot{M}_{\text {halo } \mathrm{RGs}}=1.37 \times$ $10^{-5} M_{\odot} \mathrm{kpc}^{-2} \mathrm{yr}^{-1}$.

In addition, a globular cluster loses on average about $10^{43}$ atoms $\mathrm{s}^{-1}$ (de Boer 1985) equalling a total of $4 \times$ $10^{-7} M_{\odot} \mathrm{kpc}^{-2} \mathrm{yr}^{-1}$ for the $\simeq 150$ Milky Way clusters. This GC RG mass loss adds only $0.5 \%$ to the field star value. The total is thus

$\dot{M}_{\text {total halo RGs }} \simeq 1.4 \times 10^{-5} M_{\odot} \mathrm{kpc}^{-2} \mathrm{yr}^{-1}$.

This mass loss is metal-poor.

\subsubsection{Thick disk contribution to halo RG mass loss}

In addition, the RG stars of the thick disk lose mass. Performing the same calculation as for the extended halo component one arrives with Eq. (6) for the thick disk at

$\dot{M}_{\text {total thick disk RGs }} \simeq 5.4 \times 10^{-5} M_{\odot} \mathrm{kpc}^{-2} \mathrm{yr}^{-1}$,

mass clearly lost in the lower halo, at $1<z<3 \mathrm{kpc}$. It has a metallicity between somewhat below solar and low metallicity like that of the halo.

\subsubsection{Grand total of halo RG mass loss, uncertainties}

The sum of both mass loss rates, being the mass lost by all RG stars at $z>1 \mathrm{kpc}$, material which falls toward the MW disk, equals $\dot{M}_{\text {total RG }} \simeq 7 \times 10^{-5} M_{\odot} \mathrm{kpc}^{-2} \mathrm{yr}^{-1}$.

The uncertainties in the mass loss rates $\dot{M}_{\text {RGs }}$ (Eqs. (7) and (8)) can be estimated. The value of $n_{0 \mathrm{sdB} \text { disk }}$ has an uncertainty of $30 \%$, that of the ratio of disk to halo sdB stars of about $25 \%$. The uncertainty in $h_{z}$ is $15 \%$ for the halo and $10 \%$ for the disk population. That of $g$ (depending on $h_{z}$ ) is $3 \%$ for the halo population and perhaps up to $15 \%$ for the disk one. The ratio $f_{\text {all }} \mathrm{HB} / \mathrm{sdB}$ from the birth rates is 100 , from counts of stars in CMDs it is 10. The first procedure is more accurate but still may be unceratin by a factor of 2 . The mass lost per star $\left(M_{\text {lost }}\right)$ may be different by at most $50 \%$ from the adopted value. The mass loss rate follows from halo steady state and the welldefined HB phase life. In all, this means a total uncertainty of a factor $\simeq 5$ (for each population).

\section{Observed infalling gas}

The infall rate of halo gas through HVCs has been calculated by a few authors. Oort $(1966,1970)$ derived a value of $\simeq 3 \times$ $10^{18}$ atoms $\mathrm{cm}^{-2} \mathrm{Myr}^{-1}$ from early $21 \mathrm{~cm}$ data. This translates to close to $10^{-2} M_{\odot} \mathrm{kpc}^{-2} \mathrm{yr}^{-1}$.
Wakker et al. (1999) calculate the infall rate for just Complex $\mathrm{C}$ (if at $z=5 \mathrm{kpc}$ ) as $\simeq 3 \times 10^{-3} M_{\odot} \mathrm{kpc}^{-2} \mathrm{yr}^{-1}$. Complex $\mathrm{C}$ is the biggest HVC present today covering $\simeq 4 \%$ of the sky. Considering the entire sky one can define a filling factor, $f_{\text {fill }}$, for high-velocity gas. Its value depends on the column density limit chosen. A reasonable value is $f_{\text {fill }} \simeq 0.1$ for $N_{\mathrm{H}}>10^{18.5} \mathrm{~cm}^{-2}$ (see the reappraisal of the all-sky coverage by Wakker 2004). Thus a very uncertain extrapolation of Complex $\mathrm{C}$ to the entire sky (surface area covered, area of $\mathrm{MW}$, filling factor) might mean for all $\mathrm{HV}$ gas $M_{\text {infall } \mathrm{HVC}} \simeq$ $1 \times 10^{-4} M_{\odot} \mathrm{kpc}^{-2} \mathrm{yr}^{-1}$.

Such calculations are heavily biased by choices made for distances, space velocities, and densities of halo clouds. The velocities detected are essentially LSR velocities, and a distinction in HVCs and IVCs remains arbitrary with respect to the true space motion of the gas complexes, also because there are clear signs that halo gas does not co-rotate with the disk (de Boer \& Savage 1983; Kaelble et al. 1985). The velocities themselves do not reveal much about the location of the gas. Moreover, the Magellanic Stream is not part of the general infall but can be accounted for as a special case with well-known origin.

Clearly the "observed" infall rate is very uncertain because of the imponderable space velocities and distances. As long as no better data (especially distances) are available, there is little hope for improving the estimates of the amount of infall of gas from the halo.

\section{Discussion}

The RG stars in the halo of the Milky Way lose a considerable amount of mass which is calculated in units equivalent to infall. The amount derived is a sizeable fraction of the amount estimated for the observed $\mathrm{H}$ I gas falling in from the halo. Both estimates, of the total RG mass loss and of the detected infall, are quite uncertain.

Yet, the metallicities as derived from the various UV absorption spectroscopy studies are of relevance for the discussion.

The intermediate velocity gas is relatively metal-rich (Savage \& de Boer 1981; Richter et al. 2001) while the gas of Complex C is metal-poor (Richter et al. 2001; Collins et al. 2003). The intermediate velocity gas is thought to be not far from the disk and could include gas of the galactic fountain. As shown above, also the RG stars of the lower halo will contribute gas with metallicity probably not far below solar.

The more distant parts of the halo are the regions where metal-poor halo RG stars lose their mass. It is to be expected that the metal-poor gas shed at such distances will, after some time, condense and assemble in denser pockets which eventually fall toward the disk. Given the origin at larger $z$, a higher velocity of the downflow would be expected after an appropriate time.

Other sources of material will contribute gas to the infall, too. The galactic fountain contributes metal-rich gas, while a portion of infalling gas is metal-rich Magellanic Stream gas and perhaps some gas from other accreted dwarf galaxies of unknown but likely lower metallicity. These three sources 
(RG stars, galactic fountain, dwarf galaxies) eliminate the need for substantial infall of "pristine" (zero metal) matter from intergalactic space.

Note that in the past the halo RGs were stars with larger $M_{\text {init }}$. Then $\overline{M_{\text {lost }}}$ must have been larger than today (see Weidemann 2000). But given the accepted shape of the initial mass function, their number must have been smaller and the total infall from RG mass loss was therefore not much different from that of today. Investigation of such effects is, given all uncertainties, beyond the scope of this paper.

Acknowledgements. I thank the referee Bart Wakker for probing questions about this research which stimulated an extension of the original analysis, Uli Heber for enlightening discussions, and Martin Altmann, Michael Hilker, and Philipp Richter for critical readings of the manuscript.

\section{References}

Altmann, M., Edelmann, H., \& de Boer, K. S. 2004, A\&A, 414, 181

Audouze, J., \& Tinsley, B. M. 1976, ARA\&A, 12, 43

Blitz, L., Spergel, D. N., Teuben, P. J., Hartmann, D., \& Burton, W. B. 1999, ApJ, 514, 818

Bluhm, H., de Boer, K. S., Marggraf, O., \& Richter, P. 2001, A\&A, 367,299

Bregman, J. N. 1980, ApJ, 236, 577

Charbonnel, C., Meynet, G., Maeder, A., \& Schaerer, D. 1996, A\&AS, 115,339

Chiba, M., \& Beers T. C. 2000, AJ, 119, 2843

Collins, J. A., Shull, J. M., \& Giroux, M. L. 2003, ApJ, 585, 336

de Boer, K. S. 1985, A\&A, 142, 321

de Boer, K. S., \& Savage, B. D. 1983, ApJ, 265, 210

de Boer, K. S., Aguilar Sánchez, Y., Altmann, M., et al. 1997, A\&A, 327,587
Downes, R. A. 1986, ApJS, 61, 569

Evans, A., Stickel, M., van Loon, J. Th., et al. 2003, A\&A, 408, L9

Freeman, K., \& Bland-Hawthorn, J. 2002, ARA\&A, 40, 487

Gringel, W., Barnstedt, J., de Boer, K. S., et al. 2000, A\&A, 358, L37

Heber, U. 1986, A\&A, 155, 33

Helmi, A., \& White, S. D. M. 1999, MNRAS, 307, 495

Kaelble, A., de Boer, K. S., \& Grewing, M. 1985, A\&A, 143, 408

Moehler, S., Heber, U., de Boer, K. S. 1990, A\&A, 239, 265

Muller, C. A., Oort, J. H., \& Raimond, E. 1963, CR Acad. Sci. Paris, 257,1661

Oort, J. H. 1966, Bull. Astron. Inst. Neth., 18, 421

Oort, J. H. 1970, A\&A, 7, 381

Origlia, L., Ferraro, F. R., Fusi pecci, F., \& Rood, R. T. 2002, ApJ, 571,458

Pagel, B. E. J. 1997, Nucleosynthesis and chemical evolution of galaxies (Cambridge Univ. press)

Richter, P., de Boer, K. S., Widmann, H., et al. 1999, Nature, 402, 386

Richter, P., Sembach, K. R., Wakker, B. P., \& Savage, B. D. 2001, ApJ, 562 , L181

Schmidt, M. 1968, ApJ, 151, 393

Savage, B. D., \& de Boer, K. S. 1981, ApJ, 243, 460

Savage, B. D., \& Sembach, K. R. 1996, ARA\&A, 34, 279

Schaller, G., Schaerer, D., Meynet, G., \& Maeder, A. 1992, A\&AS, 96, 269

Shapiro, P. R., \& Field, G. B. 1976, ApJ, 205, 762

Villeneuve, B., Wesemael, F., Fontaine, G., Carigan, C., \& Green, R. F. 1995, ApJ, 446, 646

Wakker, B. P. 1991, A\&A, 250, 499

Wakker, B. P. 2001, ApJS, 136, 463

Wakker, B. P. 2004, in High Velocity Clouds, ed. van Woerden et al., in preparation

Wakker, B. P., \& van Woerden, H. 1997, ARA\&A, 35, 217

Wakker, B. P., Howck, J. C., Savage, B. D., et al. 1999, Nature, 402, 388

Weidemann, V. 2000, A\&A, 363, 647

Yanny, B., Newberg, H. J., Kent, S., et al. 2000, ApJ, 540, 825 